This item was submitted to Loughborough's Research Repository by the author.

Items in Figshare are protected by copyright, with all rights reserved, unless otherwise indicated.

\title{
Into the fire: Applying Rational Emotive Behavioral Coaching (REBC) to reduce irrational beliefs and stress in fire service personnel
}

\section{PLEASE CITE THE PUBLISHED VERSION}

https://doi.org/10.1037/str0000228

\section{PUBLISHER}

American Psychological Association

\section{VERSION}

AM (Accepted Manuscript)

\section{PUBLISHER STATEMENT}

CAmerican Psychological Association, 2021. This paper is not the copy of record and may not exactly replicate the authoritative document published in the APA journal. The final article is available, upon publication, at: https://doi.org/10.1037/str0000228.

\section{LICENCE}

CC BY-NC-ND 4.0

\section{REPOSITORY RECORD}

Wood, Andrew, Andrew Wilkinson, Martin Turner, Cheryl Haslam, and Jamie Barker. 2021. "Into the Fire: Applying Rational Emotive Behavioral Coaching (REBC) to Reduce Irrational Beliefs and Stress in Fire Service Personnel". Loughborough University. https://hdl.handle.net/2134/13643186.v1. 
Running Head: REBC and Fire Service

Into the Fire: Applying Rational Emotive Behavioral Coaching (REBC) to reduce Irrational

Andrew G. Wood ${ }^{1 *}$, Andrew Wilkinson ${ }^{2}$, Martin J. Turner ${ }^{1}$, \& Cheryl O. Haslam² Jamie B. Barker ${ }^{2}$

Author Affiliations (at the time the research was done):

${ }^{1}$ Faculty of Heath, Psychology, and Social Care, Manchester Metropolitan University,

${ }^{2}$ School of Sport, Exercise and Health Sciences, Loughborough University, United Kingdom.

\section{Author Footnotes}

*Corresponding author: andrew.wood@mmu.ac.uk Faculty of Heath, Psychology, and Social Care,

Manchester Metropolitan University, United Kingdom. (The author has changed institutes since the original data collection phase was completed). Prior to publication initial findings from the manuscript were presented at an internal institutional research conference. 
Running Head: REBC and Fire Service

28 The authors would like to thank the county fire service staff, in particular the health, fitness and

29 wellbeing team led by Fiona Prew who was central to the organization and completion of this study.

30 Declarations of Interest

31 The authors report no declarations of interest. The authors alone are responsible for the content and 32 writing of this article. 


\section{Running Head: REBC and Fire Service}

\section{Abstract}

There is a scarcity of literature that reports the effects of psychological interventions to help members of the fire service operate amongst a plethora of work-related stressors. Recently, Rational Emotive Behavior Coaching (REBC) has been established as an efficacious approach to foster psychological well-being in performance contexts (e.g., elite sport, business, emergency services). Our present study is the first to explore the effects of REBC on subjective and objective (biomarkers) markers aligned with psychological well-being with a specialized and hard-to-access population of fire service personnel. Using a between groups (experimental vs. control groups) pretest-posttest field design, the immediate and maintained effects of an individualized one-to-one REBC training program (over a 12-week period) were examined on irrational performance beliefs, resilience, chronic stress (i.e., hair cortisol concentration; HCC), emotional distress and presenteeism in fire service personnel. Data showed that REBC brought about maintained reductions in irrational performance beliefs. Social validation data also indicated that REBC helped participants to better overcome adversities within and external to the workplace. Results suggested that REBC did not have a meaningful effect on HCC, symptoms of depression, anxiety and presenteeism. The efficacy of REBC as an evidence based and theoretically driven psychological framework to facilitate psychological well-being for those operating in the emergency services and more broadly extreme occupational settings are discussed. 


\section{Running Head: REBC and Fire Service}

Into the Fire: Applying Rational Emotive Behavioral Coaching (REBC) to reduce Irrational

\section{Beliefs and Stress in UK Fire Service Personnel}

The role of a fire fighter is considered an arduous occupation due to the physical demands, exposure to dangerous situations (e.g., medical crises, hazardous material, disasters), strenuous work, organizational stressors (e.g., restructures, economic uncertainty, redundancies, rotating shift patterns), and a threat to life (Poston et al., 2011). Subsequently, firefighters are at a heightened risk of developing a range of mental health issues that range from Post-Traumatic Stress Disorder (PTSD), depression, anxiety, and stress (Haslam \& Mallon, 2003). Beyond that of critical incidents, emergency personnel are also exposed to an ever-changing public-sector within the UK. Given government funding, public services, per se have seen a significant reduction in recent years (Murphy \& Ferry, 2018), and fire service personnel are required to do more with limited resources. Notably, in a House of Commons debate, a report produced by the mental health charity 'MIND' showed fire service personnel are twice as likely (compared to the wider workforce) to identify problems at work as the main cause of their mental distress.

Recognizing the substantial demands faced by UK fire-service personnel, the ability to overcome adversity has significant ramifications for one's psychological and physical health, operational functioning, and public safety. Given the specialized training required for fire service personnel, employees are difficult to replace, and more likely to experience presenteeism (i.e., showing up for work when ill and/or a loss of productivity; Johns, 2010). Excluding research in post-traumatic stress (e.g., Haslam \& Mallon, 2003) there is scant empirical literature that reports the effects of psychological interventions that re- and/or pro-actively support fire-fighters to effectively cope with the daily demands and stressors typical of their role (e.g., Aisbet et al., 2012). One psychological intervention within which enhanced human functioning is a fundamental aim, is Rational Emotive Behavior Therapy (REBT; Ellis, 1957). REBT is a cognitive-behavioral psychotherapy that focuses on the role of cognitions, emotions, and behavior in the development and maintenance of mental health (Dryden \& Brach, 2008). Though underpinned by an identical 


\section{Running Head: REBC and Fire Service}

model, the application of REBT in non-clinical settings has been retitled as 'Rational Emotional Behavior Coaching' (REBC; Kodish, 2002; Turner, 2019). Based upon a situational ABC(DE) model, REBC is centered on the premise people experience undesirable activating events (A; or adversity), about which they have rational and irrational beliefs (B; David et al., 2005). In response to an 'A', these beliefs determine the functionality (i.e., how helpful they are to one's goals) of the subsequent cognitive, emotional, behavioral, and physiological consequences (C). That is, irrational beliefs about adversity (e.g., failure, rejection and ill-treatment) lead to unhealthy negative emotions (e.g., anxiety, depression, guilt) and dysfunctional behaviors. Whereas rational beliefs (RBs) about adversity lead to healthy negative emotions (e.g., concern, sadness, remorse) and functional behaviors (Dryden \& Branch, 2008).

Irrational beliefs are positively associated with emotional distress (e.g., anxiety, depressive symptoms; David et al., 2005; Turner et al., 2019), and its effectiveness of REBC is supported with both clinical and non-clinical populations (e.g., Visla et al., 2016). Within occupational settings the application of REBC has received increasing interest (David \& Szamoskozi, 2011; Jones et al., 2020) and has been put forth as an elegant approach by directly targeting irrational beliefs that allow the client to not only 'feel better' but also 'get better' (David \& Szamoskozi, 2010; Ellis, 1994). REBC presents a model of human functioning, and beyond that of occupational settings the positive effects of REBC have been reported within education (e.g., Banks \& Zionts, 2009), military (Jarrett, 2013), and elite sport contexts (e.g., see Turner, 2016a for a review). In sum, the process of REBC posits a rational view of adversity, in turn propagating functional emotions and behaviors that facilitate goal achievement (Dryden \& Branch, 2008), all of which helping fire service personnel to better manage stress.

Researchers have highlighted conceptual similarities between REBC and the concept of resilience (e.g., Dryden, 2007; Turner, 2016b). Resilience is considered a process of coping and adapting in the face of acute or chronic adversity, whereby experiencing emotional distress is a central component of becoming resilient (Connor, 2006). In adjunct, researchers have developed 


\section{Running Head: REBC and Fire Service}

129 psychometric tools (e.g., 10-item Connor Davidson Resilience Scale; Campbell-Sills \& Stein, 2007)

130 to measure both resilient behaviors (i.e., ability to experience adversity, ability to cope with and

131 adapt to adversity, positive adaptation as a consequence of stress) and qualities (i.e., achieve goals,

132 see the humorous side, think clearly under pressure; Gonzalez et al., 2016). The application of

133 REBC encourages clients to respond to adversity with healthy negative emotions and adaptive

134 behaviors, which ultimately help rather than hinder goal achievement. REBT theory also denotes

135 when faced with adversity, rational and irrational beliefs determine healthy vs. unhealthy

136 consequences, rather than positive vs. negative response(s). To this end, REBC places emphasis on

137 adaptivity and the notion that a resilient response(s) is not absent of negatively valanced affective

138 states, but instead resilience is likely to involve considerable emotional distress (Connor, 2006;

139 Dryden, 2007). In our present study, a resilient response to adversity is considered a rational

140 response.

Research in stress management and the emergency services, although scant, is emerging

142 (e.g., Denkova et al., 2020; Jones et al., 2020). With a turn towards prevention and fostering

143 psychological wellbeing in at-risk populations (e.g., emergency services, military), improved

144 understanding of the psychological factors that bolster psychological wellbeing is directly

145 transferable to large organizations and their employees (DeTerte \& Stephens, 2014). Given the

146 centrality for effective functioning and psychological well-being in performers (Turner, 2016a),

147 researchers have called for approaches that foster resilience and coping which include the reduction

148 of catastrophic thinking, managing counterproductive beliefs, problem solving, and strengthening

149 relationships (Fletcher \& Sarkar, 2013). Indeed, more resilient fire service personnel are better able

150 to experience and garner the appropriate emotional response (both positive and negative) to match

151 the situational demands (Reynaud et al., 2013); all of which are captured within the intervention

152 aims of REBC (i.e., countering dysfunctional beliefs, enhancing anti-awfulizing, adaptive response

153 to adversity, unconditional self and other acceptance; Turner, 2016a). To begin the exploration

154 between REBC, resilience and improved performance, researchers have used the domain of elite 


\section{Running Head: REBC and Fire Service}

sport as an analogous context to establish REBC as an efficacious psychological intervention with elite performers (e.g., Wood et al., 2017a; see Turner, 2016 for an overview). While findings are emerging and appear promising, given the dearth of research with personnel in extreme occupational environments, the application of REBC warrants further examination.

Fire service personnel are required to contend with both immediate (e.g., critical incidents) and chronic stressors (e.g., organizational demands, shift work). The extant literature has largely favored the examination of acute stress (i.e., PTSD; Bryant \& Guthrie, 2007), which is surprising considering $85 \%$ of fire service personnel are said to experience chronic stress and poor mental health at work (House of Commons, 2018). In addition, although the positive effects of REBC interventions on acute performance situations are established (see Turner, 2016), less is understood about the long-term and maintained effects on objective biomarkers. To date and though limited, researchers have shown irrational beliefs to be positively associated with inflammation (i.e., increased C-reactive proteins, interleukin - 6, and increased white blood cell count; Papageorgiou et al., 2006), while reductions in irrational beliefs have been associated with lower levels of resting systolic blood pressure (Wood et al., 2017b).

Traditional methods to assess long-term endogenous markers (e.g., saliva, urine) of cortisol can be pragmatically difficult (e.g., repeated measures, Sauve et al., 2007), and limited by the sensitivity to acute stress, diurnal variation and the ethical challenge of placing too much burden on participants. More recently the analysis of hair cortisol concentration (HCC) has been put forward as a novel, pragmatic and non-invasive biomarker of chronic stress (Russell et al., 2012), which has been linked to abnormal Hypothalamus-Pituitary-Adrenal (HPA) activity (Gidlow et al., 2016). Indeed, cortisol is an end-product hormone of the HPA axis activity that proliferates in response to psychological and physical stress (Hellhammer et al., 2009). Studies have demonstrated that HCC is linked with an increased risk of cardiovascular disease (Manenschijn et al., 2013), anxiety (Steudte et al., 2011), depression (Dettenborn et al., 2012), and with stressors that interfere with circadian rhythm (Manenschijn et al.,). Though within its infancy, the assessment of HCC might offer an 


\section{Running Head: REBC and Fire Service}

181 objective biomarker with potential to improve our understanding into the long-term effects of

182 REBC on resilience and stress in the context of firefighting.

Our present study offers a comprehensive and individualized (one-to-one) applied

184

examination into the nuanced effects of REBC using both subjective and objective markers within a specialized, under represented, and hard to access population sample. In our study, we first examined the effects of REBC on psychological (i.e., irrational performance beliefs, self-reported resilience, emotional states), behavioral (i.e., presenteeism), and biological markers (hair cortisol) within members of a UK fire service. Based on current researchers (Turner, 2016a f) we hypothesized participants in the intervention group would report reductions in irrational beliefs, presenteeism, emotional states (i.e., depression, anxiety, and stress) and increases in resilience across baseline and post-intervention time-points. We also hypothesized that within the intervention group, changes in outcomes would be maintained at a 3-month time-point. Second, we sought to explore changes in biological markers of stress, as indicated by cortisol concentration taken between baseline and post-intervention time points. Finally, we sought to explore changes in outcome measures between time-points.

\section{Participants}

\section{Method}

Using a pragmatic and opportunistic sampling approach thirty-four members from a UK county Fire and Rescue service volunteered to participate in the present study (5 females, 29 males; Mage $=42.39, S D=8.06$, range $=27-59)$. Of the 34, there were 18 Firefighters, 6 crew managers, 7 watch managers, 2 station managers, and 1 group manager. All were in, or had actively served as front-line fire fighters, as such participation reflected the diversity and the range of positions within the service (Haslam \& Mallon, 2003). Of the 34, 19 participants held whole-time positions, 6 were retained (part-time), 9 held both whole and retained roles, and experience ranged from 0.25 to 38 years $(M=14.05, S D=9.25)$. Pre-screening procedures confirmed that participants had no history of/existing mental health condition and no experience of psychological support related to REBC. Institutional ethical approval and participant consent was obtained prior to the data collection. 


\section{Running Head: REBC and Fire Service}

\section{Research Design}

A between-groups pretest-posttest design was used to explore the effects of a comprehensive and bespoke one-to-one REBC intervention program with members of a county Fire and Rescue service. The intensive study of fewer subjects allowed for the application and examination of a tailored REBC intervention and nuanced participant responses. In addition, the disputation and replacement of one's core beliefs presented a challenging therapeutic process that benefits from individualized psychological provision (Dryden \& Neenan, 2015). All measures apart from social validation data were collected from all participants within each group at three different time-points, each separated by a period of three-months (i.e., baseline, post-intervention, and 3-month followup). Upon recruitment, participants were allocated to either the experimental (REBC intervention; $n$ $=18$ ) or control group (no intervention; $n=16$ ), depending on their attachment to a fire station. Similarly to previous studies (e.g., Haslam et al., 2019), the purposeful grouping of participants across county fire stations was implemented for two reasons. First, to reduce the likelihood of cross contamination of information pertaining to the one-to-one REBC intervention. That is, participants in the experimental group were unable to directly share and discuss elements and/or resources with participants in the control group (e.g., Haslam et al., 2019). Second, purposeful allocation was pragmatically informed due to the labor intensive nature of the intervention delivery and the time constraints afforded by the fire service to complete the research project. After data were collected at baseline, participants allocated to the experimental group received a tailored one-to-one REBC intervention (delivered weekly/bi-weekly) up until the post-intervention time point (12 weeks later), while participants within the control group received no formal REBC support up until the postintervention time point. After an additional 12-week period, data were collected from all participants at the 3-month follow-up time point.

\section{Measures}

Irrational performance beliefs. The irrational Performance Beliefs Inventory (iPBI; Turner et al., 2016) comprised 28-items and was used as an occupational specific assessment of the four 


\section{Running Head: REBC and Fire Service}

core irrational beliefs (demandingness, awfulizing, low-frustration tolerance, $\&$ self-depreciation) central to REBT theory. A composite score was also calculated for all core beliefs. Cronbach's alpha coefficients for the iPBI indicated internal reliability scores between $.76-.88$. The iPBI has also demonstrated construct (alpha reliability between $.91-.96$ ), concurrent (medium to large correlations), and predictive validity in a professional working environment (Turner et al., 2016). Participants were asked to indicate the extent to which they agreed on a 5-point Likert-scale between 1 (strongly disagree) to 5 (strongly agree), where higher scores indicated stronger beliefs. Resilience. The 10-item Connor Davidson Resilience Scale (CD-RISC-10; Campbell-Sills $\&$ Stein, 2007) was used to assess resilience. The CD-RISC -10 contains items that pertains to one's ability to manage and overcome challenging situations (e.g., change, personal problems, pressure, failure) and has been recommended for use within intervention studies (Windle, Bennett, \& Noyes, 2011). Cronbach's alpha coefficients for the CD-RISC-10 indicated internal reliability scores between $.75-.86$. Broadly, the CD-RISC-10 has shown high levels of internal consistency (alpha reliability between $.70-.95$ ) and construct validity (minimum of $75 \%$ of results in accordance with hypotheses; Windle et al., 2011). Participants were asked to indicate the extent to which they agreed on a 5-point Likert-scale ranging from 0 (not at all) to 4 (true nearly all of the time), where higher scores indicated increased resilience.

Hair cortisol. Hair cortisol concentration (HCC) was collected as a biological marker of chronic psychosocial stress (Gao et al., 2015). Using scissors, a hair sample of $3 \mathrm{~cm}$ adjacent to the scalp was taken from the posterior vertex position of the participant's scalp. The hair samples were tied together, wrapped in aluminum foil and stored at room temperature. Average hair growth rate of $1 \mathrm{~cm}$ per month (Wennig, 2000) was used to justify the hair sample length and the time lapse between time-points one, two and three. To this end, each sample reflected the amassed cortisol secreted over a three-month period, where higher levels of HCC reflected higher chronic stress (e.g., Gao et al., 2013) and were analyzed in blinded fashion. 


\section{Running Head: REBC and Fire Service}

Emotional distress. The Depression Anxiety Stress Scale - 21 (DASS-21; Lovibond \&

260 Lovibond, 1995) was used as a 21-item assessment of participant's negative affect (i.e.,

261 psychological wellbeing). Specifically, the DASS-21 includes the three dimensions of depression

262 (i.e., loss of self-esteem/incentives and depressed mood), anxiety (i.e., fear and anticipation of

263 negative events), stress (i.e., persistent state of over-arousal and low-frustration tolerance). In the

264 present study the DASS-21 reported high levels of internal consistency (alpha reliability between

$265.81-.93$ ), in addition demonstrating validity in clinical and non-clinical populations (Szabo, 2010).

266 Participants were asked to indicate the extent to which the statement applied to them over the past

267 week on a 4-point Likert-scale ranging from 0 (never) to 3 (almost always), where higher scores

268 indicated an increased negative emotional state for the three dimensions of depression, anxiety, and 269 stress.

Presenteeism. The World Health Organization Health and Performance Questionnaire

(HPQ; Kessler et al., 2004) was used as a self-reported measure of work performance. Participants

were asked to rate their overall work performance during the past four weeks using a 10-point

Likert-scale ranging from 0 (worst possible work performance) to 10 (top work performance). Prior

to administering this single and global measure, memory priming questions were asked to increase the response accuracy of the global rating item. For example, participants were asked to globally rate the average workers performance and their own usual performance in their job before rating their recent performance score out of 10 . To assuage between participant differences, presenteeism was calculated as a relative score whereby participants' recent own performance scores were divided by the performance of other workers. The HPQ presenteeism scale is shown to be valid and 280 reliable (test - retest .73) measure, and sensitive to change (Kessler et al., 2004). Higher scores on 281 the presenteeism score reflected greater work-related productivity in relation to the performance of most workers in the same job.

Social validation. To establish the practical (applied) effectiveness of the intervention, a 


\section{Running Head: REBC and Fire Service}

experimental group $(n=10)$. Eight participants were unable to complete the questionnaire due to other commitments at the post-intervention phase. In-line with current approaches to collecting social validation data, (e.g., Turner \& Davis, 2018) the self-reported measure used a range of open and closed (Likert-scale ranging from 1 [strongly disagree] to 7 [completely agree]) questions and was used to ascertain participants' perception of the intervention delivery and effects (Page \&

Thelwell, 2013).

\section{Data collection}

Excluding social validation data, all self-report measures (i.e., irrational performance beliefs, resilience, emotional distress) and measures of cortisol concentration were collected at baseline (month 1), post-intervention (month 4), and 3-month follow-up (month 7). In addition, participants in the experimental condition were asked to complete the social validation measure immediately after the 3-month follow-up. The start of data collection process with each participant was staggered and lasted in total a period of 9-months, with the overall data collection lasting 18months. Due to this stagger, those in in the intervention group received the REBC programme at different chronological times-points strengthening the internal validity of the present study

(Kratochwill et al., 2014).

\section{REBC Intervention}

The intervention was delivered by the lead author, a qualified REBC practitioner (Primary Practicum), and Health and Care Professions Council Registered Practitioner Psychologist in the UK. Participants in the intervention group each received an REBC program consisting of between four to six one-to-one sessions $(M=4.76, S D=.66)$ and four inter-session tasks, delivered on a weekly or fortnightly basis. One or two-week intervals were considered appropriate to maintain session momentum, whilst affording the participant opportunity to engage in the inter-session 308 (homework) tasks (Dryden \& Neenan, 2015). Each session ranged between 25 and 60 minutes in 309 length $(M=41.11, S D=8.84)$ and between 120 and 310 minutes in total intervention duration $(M=$ 194.41, $S D=51.32$ ). The overall intervention dose which participants received within this study is 


\section{Running Head: REBC and Fire Service}

311 considered comprehensive and in-line with recommendations to bring about effective REBC

312 outcomes in applied settings (Turner \& Barker, 2014). The intervention was separated into the three 313 typical phases of REBC delivery which included education, disputation, and reinforcement and 314 guided by the ABCDE framework (see Tuner \& Barker, 2014).

Procedural fidelity. This is a critical indicator of study quality in empirical and applied research, accordingly adherence to the treatment fidelity was accomplished by the use an

317 intervention manual (Tate et al., 2016). Specifically, to ensure the REBC program was a) delivered 318 consistently, b) received as intended, c) and that participants engaged with the intervention tasks, a 319 session-by-session REBC intervention guide was created (Dryden \& Branch, 2008). At the end of 320 each session, participants were encouraged to verbalize their understanding of the session content 321 and assigned the respective inter-session task. At the onset of each session, the practitioner and participant reviewed the previous session, whilst reflecting on the inter-session task, which in turn 323 influenced the content of the current session.

\section{Data Analysis}

Statistical analysis. Statistical analyses were separated into three sections. First, a series of mixed design analyses of covariance (controlled for age; ANOVA) were used to investigate the differences in irrational performance beliefs, resilience, presenteeism, emotional distress (i.e., symptoms of depression, anxiety, and stress) and cortisol between intervention and control groups (between groups factor), across baseline, post-intervention, and 3-month follow-up time points (within groups factor). Prior to the main analyses, data screening procedures were completed. To

331 limit the effect of outlying values, self-report data was checked for outliers. Data points with $\mathrm{z}$ 332 scores greater than two were winsorized (Kwak \& Kim, 2017) and replaced with the highest 333 untrimmed score. Data were winsorized for cases in iPBI (no outliers) CD-RISC10 $(n=3)$, DASS$33421(n=16) \mathrm{HCC}(\mathrm{n}=6)$, and presenteeism $(n=6)$. Preliminary checks for the iPBI, CD-RISC 10 and DASS-21were conducted and met parametric assumptions, ensuring that there was no violation 


\section{Running Head: REBC and Fire Service}

337 of regression slopes, and reliable measurement of the covariate. Effect size values (eta squared)

338 were interpreted in-line with guidelines presented by Cohen, (1988): $01=$ small effect, .06 =

339 moderate effect, .14 = large effect. Second, to explore the idiosyncratic variations typical of applied

340 research in performance contexts (e.g., Jones et al., 2020; Wood et al., 2018), percentage change

341 and Cohen's $d$ Effect Sizes (ES; Cohen, 1988) were calculated for each dependent variable between

342 baseline, post-intervention and 3-month follow-up time-point for each variable. Specifically,

343 reporting the mean, range, and frequency of values that aligned with or against the study

344 hypotheses. Finally, trends in the data using temporal partial correlational analysis, controlling for

345 group (i.e., experimental and control) and age were explored between the changes scores across all

346 variables between baseline and both post-intervention and 3-month follow-up time-points.

Hair sampling analysis. A total of 102 samples were obtained from 34 participants

348 (three/participant), and analyzed by the Anglia Ruskin University - Biomarkers Laboratory,

349 Cambridge, UK. All samples were analyzed in duplicates and three samples were re-tested at a

350 higher sample dilution. The cortisol results from ELISA $(\mu \mathrm{g} / \mathrm{dL})$ were adjusted for sample weight,

351 reconstitution volume, and the amount of methanol. Comparable to previous research $(\mathrm{r}=.95$;

352 Gidlow et al., 2016) the correlation between HCC duplicates was $r=1.000$. The reference range of 353 cortisol levels in the hair of healthy non-obese individuals was $17.7-153.2 \mathrm{pg} / \mathrm{mg}$ of hair with a 354 median of $46.1 \mathrm{pg} / \mathrm{mg}$. In the control group, HCC data for the baseline time-point was highly 355 skewed $(>-2.1 / 2.1)$ and leptokurtic (>-7.1/7.1; West, Finch, \& Curran, 1995; Skewness = 3.99, 356 Kurtosis $=15.98)$. Whereas, HCC data in the intervention group was highly skewed and/or 357 leptokurtic for the baseline $($ Skewness $=4.01$, Kurtosis $=16.45)$, post-intervention $($ Skewness $=$ 358 2.48), 3-month follow-up time points (Skewness = 2.40). Further, HCC data contained a significant 359 number of outliers ranging from $3.19 \mathrm{pg} / \mathrm{mg}$ to $737.21 \mathrm{pg} / \mathrm{mg}$. To this end, a $\log 10$ transformation 360 was applied, reducing the skewness and kurtosis of the HCC data (Sauve et al., 2007). Akin to 361 research that has used cortisol markers (e.g., Jones et al., 2020), outliers above -2/2 SDs were 


\section{Running Head: REBC and Fire Service}

winsorized ( $n=2$ for baseline, $n=2$ for post-intervention, and $n=2$ for 3-month follow-up timepoint). Raw data scores were retained to best represent mean scores (see Table 1).

\section{Results}

\section{Inferential analysis}

Irrational performance beliefs. A mixed design analysis of covariance adjusting for age revealed statistically significant differences in composite irrational performance beliefs between baseline, post-intervention and 3-month follow-up time points between the experimental and control groups $\left(F(2,62)=5.756, p=.005, \eta^{2}=.16\right)$. A Tukey post-hoc test revealed no statistically significant effect over time-points for the control group. While analysis indicated statistically significant decreases in irrational performance beliefs within the intervention group between baseline $(M=22.78, S D=3.09)$ and both post-intervention $(p<.001 ; M=18.18, S D=3.14)$ and 3month follow-up $(p<.001 ; M=18.81, S D=3.94)$ time points (see Table 1). No significant differences were reported between post-intervention and a 3-month follow-up $(p=1.00)$ in the intervention group. A series of independent samples $t$-tests between the intervention and control group revealed no significant differences in irrational performance beliefs at baseline $(t(32)=.546, \mathrm{p}$

$<.59$ ). Instead participants in intervention group reported significantly lower scores in irrational performance beliefs at both post-intervention $(t(32)=-3.830, p=.001)$, and 3-month follow-up time-points $(t(32)=-2.484, p=.018$; see Table 1) compared to the control group.

\section{[Insert Figure 1 Here]}

Resilience. A mixed design analysis of covariance, adjusting for age revealed no statistically significant differences in measures of resilience between baseline, post-intervention and 3-month follow-up time points between the experimental and control groups $\left(F(2,62)=.117, p=.89, \eta^{2}=\right.$ .004 ; see Table 1).

Hair cortisol concentration. A mixed design analysis of covariance, adjusting for age revealed no statistically significant differences in measures of log10transformed HCC data between 


\section{Running Head: REBC and Fire Service}

baseline, post-intervention and 3-month follow-up time points between the experimental and control groups $\left(F(2,62)=.750 p=.54, \eta^{2}=.020 ;\right.$ see Table 1$)$.

Psychological distress. A series of mixed design analyses of covariance, adjusting for age were conducted to examine changes in symptoms of depression, anxiety, and stress between conditions, across baseline, post-intervention and 3-month follow-up time points. Data analysis revealed no statistically significant differences in depression $\left(F(2,62)=.825, p=.44, \eta^{2}=.026\right)$ and anxiety $\left(F(2,62)=2.025, p=.141, \eta^{2}=.061\right)$, and stress $\left(F(2,62)=.448, p=.64, \eta^{2}=.014\right.$; see Table 1).

Presenteeism. After adjusting for age, a mixed design analysis of covariance revealed no statistically significant differences in relative presenteeism between baseline, post-intervention and 3 -month follow-up time points between the experimental and control groups $(F(2,62)=1.223, p$ $=.301, \eta^{2}=.038$; see Table 1$)$.

In sum, data analyses showed that participants in the REBC group reported significant reductions in irrational beliefs between baseline, post-intervention, and 3-month follow-up time points. However, data indicated no statistically significant changes in resilience, hair cortisol concentration, psychological distress and presenteeism.

\section{[Insert Table 1 Here]}

\section{Effect size and percentage change.}

In Table 2 percentage changes scores, as well as frequency of effect sizes that aligned with, or went against the study hypotheses have been detailed between baseline, post-intervention, and 3month follow-up time points. As hypothesized data showed that compared to the control group those in the intervention group reported a greater reduction in irrational beliefs between baseline and post-intervention time-points. Reductions in irrational beliefs were maintained at a 3-month follow-up timepoint (see Table 2). Within the intervention group, data indicated incremental increases in resilience across time-points, whereby effect size calculations aligned with the hypotheses. Nonetheless, we see a similar pattern in the control group where participants indicate a 


\section{Running Head: REBC and Fire Service}

413 greater number of effect size scores that are contrary to the study hypotheses (i.e., resilience scores 414 would remain the same or reduce over the course of the study). Although HCC, depression, and 415 anxiety scores showed mean reductions in both intervention and control groups across time-points, 416 the effects size frequencies showed no general pattern of change in accordance to the study 417 hypotheses across time-points. That is, for HCC, depression and anxiety, the REBC group showed 418 no greater reductions across time-points than the control group. In the control group the frequency 419 of effect sizes for self-reported stress were aligned with the study hypotheses (i.e, maintenance and/or increases in stress between time-points). In addition, mean effect size scores indicate marked reductions in perceived stress between baseline and both post-intervention and 3-month follow-up time-points for the intervention group. Generally, those in the control group reported changes in presenteeism (i.e. productivity) scores in the hypothesized direction compared to that of the intervention group. That is, the intervention group reported incremental increases in productivity (i.e., work related performance) across time-points, however, the control group reported no pattern of change.

\section{Exploratory correlational analyses.}

Partial correlation coefficients for change scores between baseline and both postintervention and 3-month follow-up time-points were calculated for all participants whilst controlling for group (i.e., intervention and control) and age (see supplementary material for correlation matrices). Between baseline and post-intervention time-points data showed irrational performance beliefs to be negatively correlated with resilience ( $r=-.40, p=.23$ ), and positively correlated with stress $(r=.43, p=.01)$, and anxiety $(r=-.42, p=.01)$. Further, changes in stress and anxiety were positively correlated $(r=.45, p=.01)$. Between baseline and the 3 -month follow-up time-point data only showed a significant positive correlation between resilience and depression $(r$

$437=-.35, p=.049)$. Between post-intervention and 3-month follow-up the data showed a significant 438 positive correlation between irrational performance beliefs and anxiety $(r=-.35, p=.045)$. 


\section{Running Head: REBC and Fire Service}

\section{Social validation}

The quantitative social validation responses (see Table 3 ) indicated the positive effects and application of the REBC intervention. Qualitative accounts also showed nine out of ten participants ( $n=1$ left blank) reported that the one-to-one intervention program helped them feel more capable of approaching and dealing with adversities in future, beyond that of the workplace. In addition, participants reported improvements in being able to respond more adaptively to life's adversity, via greater emotional control, empathy for others, and greater perspective of negative experiences. Six participants highlighted that the intervention allowed them to take a better perspective in the face of adverse life events, which reflects the core rational belief of anti-awfulizing. When asked about the underlying reasons for the relative success/failure of the program, participants reported greater selfawareness of their own beliefs and actions, in adjunct to rational shifts that made flexible their expectations of themselves and others. Three participants cited feeling disappointed that the one-toone sessions had ceased, while 7 participants offered positive remarks regarding the intervention delivery as the sessions ended. Such comments suggested that participants may have benefited from a greater number of sessions, while appreciating that they themselves had developed and accrued the tools to overcome future challenges within and outside of work settings.

\section{[Insert Table 3 Here]}

\section{Discussion}

The present study was the first to examine the effects of REBC on irrational performance beliefs, resilience, hair cortisol concentration (chronic stress), psychological distress (i.e., symptoms of depression, anxiety, and stress), and presenteeism in members of a UK fire service. First, we hypothesized that the intervention would bring about reductions in irrational performance beliefs, psychological distress (i.e., depression, anxiety, and stress), and presenteeism; as well as, increases in resilience between baseline, post-intervention, and 3-month follow-up time points. Second, changes in biological markers of stress, as indicated by cortisol concentration were explored. Finally, partial correlational analyses were conducted on all outcome variables, across groups 


\section{Running Head: REBC and Fire Service}

investigating patterns of change between baseline, post-intervention, and 3-month follow-up timepoints.

In-line with our hypothesis, data showed only the intervention group reported significant reductions in irrational performance beliefs between baseline and post-intervention time-points. Our findings contribute to emerging literature that supports the application of REBC as an effective intervention to reduce psychologically deleterious irrational beliefs within emergency service personnel (e.g., law enforcement; Jones et al., 2020) and more broadly within non-clinical populations (e.g., organisations; David \& Szamoskzi, 2010; Turner \& Barker, 2015, elite sport; Turner \& Davis, 2018, \& exercise settings; Outar et al., 2018). In line with previous research (e.g., Wood et al., 2017a) data also showed that the application of REBC brought about acute and maintained reductions in irrational performance beliefs. Sustained reductions in irrational beliefs reflect the concept of the 'elegant solution' (Dryden \& Neenan, 2015). Whereby, the REBC intervention led to fundamental and philosophical changes in participants core beliefs that did not revert back to baseline once the intervention was withdrawn. Furthermore, after controlling for group and age, correlational analyses indicated greater endorsement of irrational performance beliefs were matched with significantly lower resilience scores and higher levels of stress and anxiety between baseline and post-intervention time-points. As such, irrespective of the intervention, these findings reinforce the positive association between irrational beliefs with markers of stress and anxiety (see Visla et al., 2016) for those working within the emergency services (i.e., fire service personnel).

These findings have significant applied implications for two reasons. First, fire service personnel are faced with a plethora of organizational, performance, and personal stressors that bring with it significant psychological and physical demands. As such, the absence and/or low endorsement of irrational performance beliefs would suggest fire service personnel who endorse greater rational alternatives will respond with healthy negative emotions and functional behaviors when faced with adversity. That is, a situation which is incongruent with what is motivationally 


\section{Running Head: REBC and Fire Service}

relevant to that individual (e.g., failure, rejection, or poor treatment). Second, given the moral and ethical obligation for organizations to adequately prepare and support fire service personnel, REBC presents a somewhat brief yet comprehensive intervention to bring about sustained reductions in irrational and harmful beliefs with those operating in extreme occupational settings. Indeed social validation data indicated that participants not only grasped the ABCDE framework central to REBC, but were also cognizant to its continued use and engagement when encountering future adversities. Ultimately, our data adds to the evidence-base that REBC is an efficacious intervention to reduce irrational beliefs for those operating in high-performance settings such as the emergency services.

In contrast to our hypothesis, participants did not report increases in resilience after receiving the REBC intervention at post-intervention or 3-month follow-up time-points. Notably, both the intervention $(M=32.11, S D=4.63)$ and the control group $(M=29.75, S D=4.31)$ recorded scores of resilience comparable to those operating in other emergency services (i.e., $n=114$; police officers; McCanlies et al., 2014). Though not statistically significant, at the 3-month follow-up time-point the intervention group $(M=34.06)$ reported higher scores on resilience compared to the control group $(M=30.50)$, and the mean scores put forth by McCanlies et al. While it would be prudent to not overstate these findings, given the centrality of adversity in the conceptualization to resilience (Fletcher \& Sarkar, 2013) it is unsurprising that participants may report greater resilience in adjunct to reductions in irrational beliefs (Dryden, 2007; Turner, 2016b). Social validation also indicated that nine out of ten participants felt more capable of approaching and dealing with adversities in the future.

In addition to previous research with athletes (e.g., Deen et al., 2017), our study is the first to suggest increases in resilience using REBC with those operating in the emergency services. Using the ABCDE framework, REBC practitioners dispute and challenge irrational beliefs about adversity to cultivate rational alternatives, and ultimately a rational philosophy. Thus, individuals operating in the fire service are better equipped to manage their beliefs regarding the various 


\section{Running Head: REBC and Fire Service}

517 adversities they encounter on a daily basis. These beliefs are salient for early career fire service

518 personnel, who are at increased odds of making a mistake in conjunction with negative self-

519 appraisals (indicative of irrational beliefs), which have been highlighted as a pre-exposure risk

520 factor for PTSD (Bryant \& Guthrie, 2007). Indeed, there exists a plethora of research that has

521 established rational beliefs about adversity (i.e., failure, rejection and setbacks) lead to adaptive

522 thoughts, emotions and behaviors that facilitate one's goal achievement (David et al., 2010).

Our study is the first to examine the effects of REBC on chronic stress via HCC in the

emergency services (i.e., fire service). Data showed no significant differences in HCC data for both

variation in the data. Nevertheless, mean scores of HCC tenuously showed that participants in the intervention group reported greater decreases in $\mathrm{HCC}$ between baseline and 3-month follow-up time point scores compared to those in the control group. Considering previous researchers have reported the links between reductions in irrational beliefs with reductions in physiological outcomes (e.g., Blood pressure; Wood et al., 2017b) and biological indicators of health (i.e., greater risk of cardiovascular ill-health; Papageorgiou et al., 2006), the measurement of biomarkers of stress remains to be a promising and objective marker for future investigations. Nonetheless, our study 533 demonstrates the pragmatic challenges of collecting markers of chronic stress using hair samples 534 and conducting statistical analyses with sufficient statistical power when undertaking a comprehensive applied investigation, with a hard to access and specialized population group. Indeed, the HCC data include greater variations in scores, which although controlled for during the 537 study (i.e., hair dye, collection of hair from vertex posterior), the impact of factors such as 538 drugs/medication on HCC are unknown (Suave et al., 2007). Furthermore, we were aware that 539 cortisol levels and negative psychological outcomes may not be positively correlated in linear 540 fashion. Researchers have shown that those extremely stressed or burned out may present with 541 hypocortisolemia, which is the blunted secretion of cortisol levels (Steudte et al., 2011). To this end, 


\section{Running Head: REBC and Fire Service}

researchers should consider HCC a non-invasive, objective, and yet complementary assessments of chronic stress.

Concerning both self-reported symptoms of depression and anxiety the data showed no statistical differences between time-points. Further, frequency of effect size calculations showed no pattern for or against the present hypothesis (i.e., participants in the intervention would report greater decreases in symptoms of depression and anxiety compared to the control group). That is, both groups displayed similar reductions in symptoms of anxiety and depression at a 3-month follow-up time-point. While the inferential analysis revealed no significant effects, the frequency of effect size displayed small increases in presenteeism between baseline and 3-month follow-up time-points, whilst the control group reported a minor reduction. On the contrary to these findings social validation data noted that participants were ambivalent to the direct and positive influence of the intervention on workplace performance. Instead, participants reported positive changes to more proximal mechanisms of change associated with shifts in their core beliefs. For example, participants ability to better overcome adversity was explained by greater emotional control, enhanced empathy for others (when in conflict), and improved perspective of negative experiences.

\section{Strengths and Limitations}

Our study compares well to many other empirical and field-based studies (e.g., David \& Szamoskozi, 2010; Jones et al., 2020), having applied a significant one-to-one intervention with a specialized and hard to reach population group. The application of the REBC intervention was tailored to each participant in the intervention group, and though plausible, the inclusion of an individualized and one-to-one attention placebo condition (e.g., Wood et al., 2018) was considered pragmatically unfeasible. Thus, in the present study we cannot completely exclude the possibility that changes in intervention group did not result from a placebo effect (Boot et al., 2013).

Due to the comprehensive and labor intensive application of REBC within a hard to reach and specialized population group, the sample size limited the statistical power of the inferential statistics. For example, HCC was used to objectively determine changes in stress, however the data 


\section{Running Head: REBC and Fire Service}

showed the REBC did not have a significant effect on pre-, post-intervention, and 3-month followup cortisol levels. Considering the data variability reported in $\mathrm{HCC}$, these findings are perhaps unsurprising and are in-line with previous researchers (e.g., Jones et al., 2020). For example, data showed a range in HCC from $4.39 \mathrm{pg} / \mathrm{mg}-143.72 \mathrm{pg} / \mathrm{mg}$ at the baseline time-point, $3.86 \mathrm{pg} / \mathrm{mg}-$ $111.23 \mathrm{pg} / \mathrm{mg}$ at the post-intervention time-point and 3.19pg/mg - 38.22 at the 3-month follow-up time-point. To address these limitations, future researchers may wish to first, repeatedly assess HCC (1cm in length) more frequently on a one monthly basis (e.g., Goldberg et al., 2014). Second, conduct a prospective cross-sectional research design with a sample size allowing for sufficient statistical power. Finally, to use urine or saliva samples that assess participants acute response to stressful situations (Sauve et al., 2007).

While maintaining the intervention fidelity and procedural reliability, our present study represents a comprehensive one-to-one application of REBC with 18 participants. Considering the shortage of follow-up measures in the literature, we attempted to document the acute and maintained effects of REBC. Given the aforementioned strengths there are methodological and research related challenges, typical of field-based studies. These include a lack of repeated assessments for each outcome measure, as well, maturation, learning, experience, and practice effects that may threaten the internal validity of a study. While we are aware of the ethical burden and tedium effect on participants when collecting repeated measurements from fewer cases (e.g., Normand et al., 2016), future researchers could adopt single-case research methods (e.g., the multiple-baseline, staggered single-case research design; Kazdin, 2019 ) to better delineate the multi-faceted nature and complexities surrounding applied psychological interventions used in conjunction with innovative biomarkers of stress, such as HCC. Such methods would enable the idiographic examination of an REBC intervention using a triangulation of subjective, objective, and behavioral markers with fewer cases (Normand et al., 2016).

According to a Home Office (2018) report, 5.12\% of serving fire service personnel were female, and though the current sample remains significantly skewed towards males the present 


\section{Running Head: REBC and Fire Service}

ratio exceeds the national representation with five of the intital 34 participants being female (14.71\%). Future researchers are recommended to consider an equal gender balance within their samples.

\section{Implications for Practice}

Our study provides valuable insights into the application and effects of REBC with fire service personnel. Members of the fire service are faced with changing operational and organizational demands, which are often outside of their immediate control. Therefore, the ABCDE framework central to REBC, offered participants a logical and coherent framework for participants to determine and control how they respond to the circumstances in which they find themselves. Specifically, REBC provided participants with a framework by which to recognize their own beliefs about adversity, and re-appraise and replace them with rational alternatives. Not limited to the typical (i.e., critical incidents) demands fire service personnel are faced with, many participants put forth challenges associated with lifestyle, personal and organizational matters. Thus, REBC brings about philosophical and meaningful change in one's view of adversity, which transcend that of the workplace. For example, someone who no longer demands, but instead strongly prefers to be treated fairly, will propagate an adaptive response to be being treated unfairly across contexts.

While, all humans are inherently irrational (David et al., 2010), it would be prudent to draw attention to the prospect of floor effects in the present study, where participants reported relatively low scores of irrational performance beliefs. Future researchers and/or practitioners should consider the process of screening personnel to prioritize those who present with a greater need and/or benefit from receiving REBC. Importantly, REBC is typically viewed as a remedial provision (e.g., dealing with problems) and typically delivered on a one-to-one basis, which can be labor intensive for practitioners. Further, $37 \%$ of fire service members perceived that colleagues would treat them negatively if they spoke about mental health issue, and were more likely to seek support from a GP than a colleague (House of Commons, 2018). Thus, future researchers should look to examine the systemic integration of principles of REBC to bring rational shifts and facilitate psychological 


\section{Running Head: REBC and Fire Service}

health for all those that operate within a system (i.e., senior leadership teams, organizational purpose, objectives and values).

\section{Conclusion}

Our study demonstrated REBC to be an effective intervention in bringing about acute and maintained reductions in the irrational performance beliefs of fire service personnel. The links between the REBC intervention and markers of anxiety, depression, and presenteeism were nonconclusive. However, descriptive data indicated that fire service personnel who received the REBC intervention, showed reductions in self-reported symptoms of stress, and were better placed to overcome any future adversities (e.g., rejection, failure, set-backs, poor-treatment). We conclude that HCC provides a non-invasive objective biomarker of chronic stress, however, data variability indicates it should, at this juncture remain a complementary measurement. Our study contributes to the emerging literature regarding the application of REBC as an promising intervention to bring about positive psychological benefits with those operating in highly demanding and performancerelated work-place settings such as the emergency services.

\section{References}

Aisbett, B., Wolkow, A., Sprajcer, M., \& Ferguson, S. A. (2012). “Awake, smoky, and hot”: providing an evidence-base for managing the risks associated with occupational stressors encountered by wildland firefighters. Applied Ergonomics, 43, 916-925. http://doi:10.1016/j.apergo.2011.12.013

Banks, T., \& Zionts, P. (2009). REBT used with children and adolescents who have emotional and behavioral disorders in educational settings: A review of the literature. Journal of RationalEmotive \& Cognitive-Behavior Therapy, 27, 51-65. http://doi:10.1007/s10942-008-0081-x

Boot, W. R., Simons, D. J., Stothart, C., \& Stutts, C. (2013). The pervasive problem with placebos in psychology: Why active control groups are not sufficient to rule out placebo effects. Perspectives on Psychological Science, 8, 445-454. http://doi. $10.1177 / 1745691613491271$ 


\section{Running Head: REBC and Fire Service}

Braig, S., Grabher, F., Ntomchukwu, C., Reister, F., Stalder, T., Kirschbaum, C., Genuneit, J., \& Rothenbacher, D. (2015). Determinants of maternal hair cortisol concentrations at delivery reflecting the last trimester of pregnancy. Psychoneuroendocrinology, 52, 289-296. http://doi.10.1016/j.psyneuen.2014.12.006

Bryant, R. A., \& Guthrie, R. M. (2007). Maladaptive self-appraisals before trauma exposure predict posttraumatic stress disorder. Journal of Consulting and Clinical Psychology, 75, 812. doi://10.1037/0022-006X.75.5.812.

Campbell-Sills, L., \& Stein, M. B. (2007). Psychometric analysis and refinement of the connordavidson resilience scale (CD-RISC): Validation of a 10-item measure of resilience. Journal of Traumatic Stress: Official Publication of The International Society for Traumatic Stress Studies, 20, 1019-1028.

Cohen, J. (1988). Statistical power analysis for the behavioral sciences (2nd ed.). Erlbaum.

Connor, K. M. (2006). Assessment of resilience in the aftermath of trauma. Journal of Clinical Psychiatry, 67, 46-49.

David, A. R., \& Szamoskozi, S. (2011). The effectiveness of a Rational Emotive Behavioral Coaching program to reduce irrationality in organizational environment and the mediating effect of modifying irrational cognitions on emotional distress and quality of life. Transylvanian Journal of Psychology, 77-93.

David, D., Szentagotai, A., Eva, K., \& Macavei, B. (2005). A synopsis of rational-emotive behavior therapy (REBC); fundamental and applied research. Journal of Rational-Emotive \& Cognitive-Behavior Therapy, 23, 175-221. https://doi:10.1007/s10942-005-0011-0.

Deen, S., Turner, M. J., \& Wong, R. S. (2017). The Effects of REBC, and the Use of Credos, on Irrational Beliefs and Resilience Qualities in Athletes. The Sport Psychologist, 1-39. https://doi.org/10.1123/tsp.2016-0057 


\section{Running Head: REBC and Fire Service}

Denkova, E., Zanesco, A. P., Rogers, S. L., \& Jha, A. P. (2020). Is Resilience Trainable? An Initial Study Comparing Mindfulness and Relaxation Training in Firefighters. Psychiatry Research, 285, 112-131. https://doi.org/10.1016/j.psychres.2020.112794

De Terte, I., \& Stephens, C. (2014). Psychological resilience of workers in high-risk occupations. Stress and Health, 30, 353-355. https://doi.org/10.1002/smi.2627

Dettenborn, L., Muhtz, C., Skoluda, N., Stalder, T., Steudte, S., Hinkelmann, K., Kirschbaum, C., \& Otte, C. (2012). Introducing a novel method to assess cumulative steroid concentrations: Increased hair cortisol concentrations over 6 months in medicated patients with depression. Stress, 15, 348-353. http://doi:10.3109/10253890.2011.619239

Dryden, W. (2007). Resilience and rationality. Journal of Rational-Emotive \& Cognitive-Behavior Therapy, 25, 213-226. https://doi: 10.1007/s10942-006-0050-1.

Dryden, W., \& Branch, R. (2008). Fundamentals of rational emotive behavior therapy: A training handbook. John Wiley \& Sons.

Dryden, W., \& Neenan, M. (2015). Rational emotive behav- iour therapy: 100 key points and techniques. Routledge.

Ellis, A. (1957). Rational psychotherapy and individual psychology. Journal of Individual Psychology, 13, 38-44.

Ellis, A. (1994). Reason and emotion in psychotherapy. Revised and updated edition. Birch Lane Press.

Fletcher, D., \& Sarkar, M. (2013). Psychological resilience. European Psychologist, 18, 1223. https://doi.org/10.1027/1016-9040/a000124.

Gonzalez, S. P., Moore, E. W. G., Newton, M., \& Galli, N. A. (2016). Validity and reliability of the Connor-Davidson Resilience Scale (CD-RISC) in competitive sport. Psychology of Sport and Exercise, 23, 31-39. https://doi.org/10.1016/j.psychsport.2015.10.005. 


\section{Running Head: REBC and Fire Service}

Goldberg, S. B., Manley, A. R., Smith, S. S., Greeson, J. M., Russell, E., Van Uum, S., \& Davis, J. M. (2014). Hair cortisol as a biomarker of stress in mindfulness. The Journal of Alternative and Complementary Medicine, 20(8), 630-634.

Lovibond, S.H. \& Lovibond, P.F. (1995). Manual for the Depression Anxiety \& Stress Scales. (2 ${ }^{\text {nd }}$ Ed.) Sydney: Psychology Foundation.

Gao, W., Stalder, T., Foley, P., Rauh, M., Deng, H., \& Kirschbaum, C. (2013). Quantitative analysis of steroid hormones in human hair using a column-switching LC-APCI-MS/MS assay. Journal of Chromatography, 928, 1-8. https://doi:10.1016/j.jchromb.2013.03.008

Gidlow, C. J., Randall, J., Gillman, J., Smith, G. R., \& Jones, M. V. (2016). Natural environments and chronic stress measured by hair cortisol. Landscape and Urban Planning, 148, 61-67. https://doi.org/10.1016/j.landurbplan.2015.12.009

Haslam, C., Kazi, A., Duncan, M., Clemes, S., and Twumasi, R. (2019). Walking Works Wonders: a tailored workplace intervention evaluated over 24 months. Ergonomics, 62: 31-41. https://doi.org/10.1080/00140139.2018.1489982.

Haslam, C., \& Mallon, K. (2003). A preliminary investigation of post-traumatic stress symptoms among firefighters. Work \& Stress, 17, 277-285. https://doi: https://doi.org/10.1080/02678370310001625649

Hellhammer, D. H., Wüst, S., \& Kudielka, B. M. (2009). Salivary cortisol as a biomarker in stress research. Psychoneuroendocrinology, 34, 163-171. https://doi.org/10.1016/j.psyneuen.2008.10.026

Home Office (2018). Fire statistics data tables. London. https://www.gov.uk/government/statistical-data-sets/fire-statistics-data-tables\#workforceand-workforce-diversity

House of Commons. (2018). Mental health support for firefighters. London. House of Commons Library.

Jarrett, T.A. (2013). Warrior Resilience and Thriving (WRT): Rational Emotive Behavior Therapy 


\section{Running Head: REBC and Fire Service}

(REBC) as a Resiliency and Thriving Foundation to Prepare Warriors and Their Families for Combat Deployment and Posttraumatic Growth in Operation Iraqi Freedom, 2005-2009. Journal or Rational-Emotive \& Cognitive Behavior Therapy, 31, 93-107.

Jones , J., Turner, M.J., \& Barker, J.B. (Accepted $3^{\text {rd }}$ of September 2020). The effects of a cognitive behavioral stress intervention on the motivation and wellbeing of senior UK police personnel. International Journal of Stress Management.

Kazdin, A. E. (2019). Single-case experimental designs. Evaluating interventions in research and clinical practice. Behaviour Research and Therapy, 117, 3-17. https://doi.org/10.1016/j.brat.2018.11.015

Kessler, R. C., Ames, M., Hymel, P. A., Loeppke, R., McKenas, D. K., Richling, D. E., Stang, P.E., \& Ustun, T. B. (2004). Using the World Health Organization Health and Work Performance Questionnaire (HPQ) to evaluate the indirect workplace costs of illness. Journal of Occupational and Environmental Medicine, 46, 23-37. https://doi: 10.1097/01.jom.0000126683.75201.c5

Kodish, S. P. (2002). Rational emotive behavior coaching. Journal of Rational-Emotive \& Cognitive Behavior Therapy, 20, 235-246.

Kratochwill, T. R., \& Levin, J. R. (2014). Enhancing the scientific credibility of single-case intervention research: Randomization to the rescue. In T. R. Kratochwill \& J. R. Levin (Eds.), School psychology series. Single-case intervention research: Methodological and statistical advances (p. 53-89). American Psychological Association. https://doi.org/10.1037/14376-003

Kwak, S. K., \& Kim, J. H. (2017). Statistical data preparation: management of missing values and outliers. Korean Journal of Anesthesiology, 70, 407-411. https://doi:10.4097/kjae.2017.70.4.407

Manenschijn, L., Schaap, L., Van Schoor, N., Van der Pas, S., Peeters, G., Lips, P., Koper, J.W., \& Van Rossum, E. (2013). High long-term cortisol levels, measured in scalp hair, are 


\section{Running Head: REBC and Fire Service}

associated with a history of cardiovascular disease. The Journal of Clinical Endocrinology \& Metabolism, 98, 2078-2083. https://doi.org/10.1210/jc.2012-3663

McCanlies, E.C., Mnatsakanova, A., Andrew, M.E., \& Burchfiel, C.M. (2014). Positive psychological factors are associated with lower PTSD symptoms among police officers: post Hurricane Katrina. Stress and Health, 30, 405-415. https:doi: 10.1002/smi.2615.

Murphy, P., \& Ferry, L. (2018). Another Turn of the Screw: Fire and Rescue Under the Coalition Government of 2010-2015. In Fire and Rescue Services (pp. 45-59). Springer.

Normand, M. P. (2016). Less is more: Psychologists can learn more by studying fewer people. Frontiers in Psychology, 7, 934 -938. https://doi.org/10.3389/fpsyg.2016.00934

Outar, L., Turner, M. J., Wood, A. G., \& Lowry, R. (2018). "I need to go to the gym”: Exploring the use of rational emotive behavior therapy upon exercise addiction, irrational and rational beliefs. Performance Enhancement \& Health, 6, 82-93. https://doi.org/10.1016/j.peh.2018.05.001

Page, J., \& Thelwell, R. (2013). The value of social validation in single-case methods in sport and exercise psychology. Journal of Applied Sport Psychology, 25, 61-71. https://doi:10.1080/10413200.2012.663859

Papageorgiou, C., Panagiotakos, D. B., Pitsavos, C., Tsetsekou, E., Kontoangelos, K., Stefanadis, C., \& Soldatos, C. (2006). Association between plasma inflammatory markers and irrational beliefs; the ATTICA epidemiological study. Progress in Neuro-Psychopharmacology and Biological Psychiatry, 30, 1496-1503. https://doi:10.1016/j.pnpbp.2006.05.018

Poston, W. S., Jitnarin, N., Haddock, C. K., Jahnke, S. A., \& Tuley, B. C. (2011). Obesity and Injury-Related Absenteeism in a Population-Based Firefighter Cohort. Obesity, 19, 20762081. http://doi:10.1038/oby.2011.147

Reynaud, E., Guedj, E., Souville, M., Trousselard, M., Zendjidjian, X., El Khoury-Malhame, M., Fakra, E., Nazarian, B., Blin, O., Canini, F., \& Khalfa, S. (2013). Relationship between emotional experience and resilience: an fMRI study in fire-fighters. Neuropsychologia, 51, 


\section{Running Head: REBC and Fire Service}

Russell, E., Koren, G., Rieder, M., \& Van Uum, S. (2012). Hair cortisol as a biological marker of chronic stress: current status, future directions and unanswered questions. Psychoneuroendocrinology, 37, 589-601.http:// doi: 10.1016/j.psyneuen.2011.09.009.

Sauvé, B., Koren, G., Walsh, G., Tokmakejian, S., \& Van Uum, S. H. (2007). Measurement of cortisol in human hair as a biomarker of systemic exposure. Clinical and Investigative Medicine, 183-191. http://doi:10.25011/cim.v30i5.2894

Steudte, S., Stalder, T., Dettenborn, L., Klumbies, E., Foley, P., Beesdo-Baum, K., \& Kirschbaum, C. (2011). Decreased hair cortisol concentrations in generalised anxiety disorder. Psychiatry Research, 186, 310-314. http://doi:10.1016/j.psychres.2010.09.002

Sauvé, B., Koren, G., Walsh, G., Tokmakejian, S., \& Van Uum, S. H. (2007). Measurement of cortisol in human hair as a biomarker of systemic exposure. Clinical and Investigative Medicine, 30, 183-191. https://doi.org/10.25011/cim.v30i5.2894

Szabó, M. (2010). The short version of the Depression Anxiety Stress Scales (DASS-21): Factor structure in a young adolescent sample. Journal of Adolescence, 33, 1-8.

Tate, R. L., Perdices, M., Rosenkoetter, U., Shadish, W., Vohra, S., Barlow, D. H., ... \& Sampson, M. (2016). The single-case reporting guideline in behavioral interventions (SCRIBE) 2016 statement. Physical Therapy, 96, 1-10.http://doi:10.1037/arc000027.

Turner, M. J. (2016a). Rational Emotive behavior therapy (REBC), irrational and rational beliefs, and the mental health of athletes. Frontiers in Psychology, 7, 1423. https://doi:10.3389/fpsyg.2016.01423.

Turner, M. J. (2016b). Proposing a rational resilience credo for use with athletes. Journal of Sport Psychology in Action, 7, 170-181. https://doi.org/10.1080/21520704.2016.1236051

Turner, M. J. (2019). REBT in Sport. In, M.E. Bernard \& W. Dryden (Eds.), Advancing REBT Theory, Research and Practice. New York: Springer. 


\section{Running Head: REBC and Fire Service}

Turner, M. J., Aspin, G., \& Gillman, J. (2019). Maladaptive schemas as a potential mechanism through which irrational beliefs relate to psychological distress in athletes. Psychology of Sport and Exercise, 44, 9-16. https://doi.org/10.1016/j.psychsport.2019.04.015

Turner, M.J., \& Barker, J.B. (2014). Using rational emotive behavior therapy with athletes. The Sport Psychologist, 28, 75-90. https://doi:10.1123/tsp.2013-0012

Turner, M. J., \& Davis, H. (2018). Exploring the effects of Rational Emotive Behavior Therapy (REBT) on the irrational beliefs and self-determined motivation of triathletes. Journal of Applied Sport Psychology, 31, 53-272. https://doi:10.1080/10413200.2018.1446472

Turner, M. J., Allen, M., Slater, M. J., Barker, J. B., Woodcock, C., Harwood, C. G., \& McFayden, K. (2016). The development and initial validation of the Irrational Performance Beliefs Inventory (iPBI). European Journal of Psychological Assessment. Advance online publication. https://doi:10.1027/1015-5759/a000314

Vîsla , A., Flückiger, C., grosse Holtforth, M., \& David, D. (2016). Irrational beliefs and psychological distress: A meta-analysis. Psychotherapy and Psychosomatics, 85, 8-15. https://doi:10 .1159/000441231.

Wennig, R. (2000). Potential problems with the interpretation of hair analysis results. Forensic Science International, 107, 5-12. https://doi.org/10.1016/S0379-0738(99)00146-2.

West, S. G., Finch, J. F., \& Curran, P. J. (1995). Structural equation modeling: concepts, issues and applications SAGE Publications. Thousand Oaks, $C A$.

Windle, G., Bennett, K.M., \& Noyes, J. (2011). A meth- odological review of resilience measurement scales. Health and Quality of Life Outcomes, 9, 8-25. https://doi:10.1186/1477-7525-9-8.

Wood, A. G., Barker, J. B., \& Turner, M. J. (2017a). Developing performance using rational emotive behavior therapy (REBC): A case study with an elite archer. Sport Psychologist, 31, 78-87. https://doi.org/10.1123/tsp.2015

Wood, A. G., Barker, J. B., Turner, M., \& Sheffield, D. (2017b). Examining the effects of Rational 
Running Head: REBC and Fire Service Emotive Behavior Therapy (REBT) on Performance Outcomes in Elite Paralympic Athletes. Scandinavian Journal of Medicine \& Science in Sports. https://doi.org/10.1111/sms.12926.

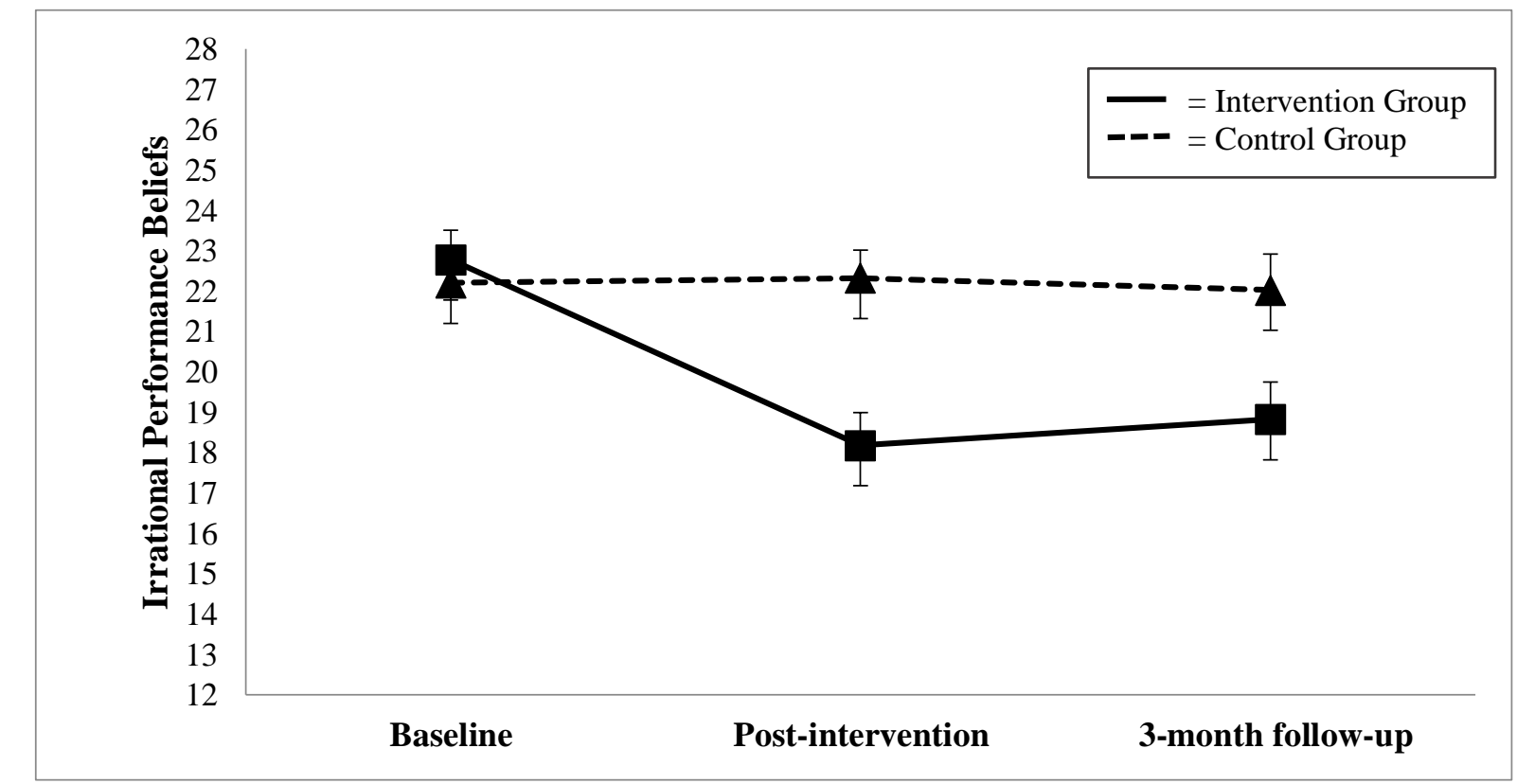

Figure 1. Composite irrational performance belief scores for the intervention group and control group at baseline, post-intervention, and 3-month follow-up time points. Standard errors are represented in the figure by error bars attached to each data point. 
Running Head: REBC and Fire Service

845 Table 1.

846 Mean and standard deviation scores for all outcome variables.

\begin{tabular}{llcccc}
\hline Group & $\begin{array}{c}\text { Baseline } \\
(M \pm \mathrm{SD})\end{array}$ & $\begin{array}{c}\text { Post- } \\
\text { intervention } \\
(M \pm \mathrm{SD})\end{array}$ & $\begin{array}{c}3 \text {-month follow-up } \\
(\text { M }\end{array}$ & $F$ SD) & $\eta^{2}$
\end{tabular}

iPBI

Intervention

22.78(3.09)

Control

$22.20(3.03)$

Intervention

$32.11(4.63)$

$33.06(3.30)$

34.06 (3.19)

$$
\text { Control }
$$

$29.75(4.31)$

$30.75(4.46)$

$30.81(3.66)$

852

\section{DASS-21}

Intervention

4.44(5.38)

$2.56(4.33)$ Depression

Control

$6.00(6.85)$

5.25(8.97)

DASS-21

Intervention

3.89(3.79)

$2.22(2.56)$ Anxiety

Control

$5.00(5.11)$

$3.00(2.89)$

$6.00(5.78)$

7.33(4.34)

DASS-21

Intervention

$10.22(6.54)$

9.63(7.24)

$8.50(5.68)$

9.13(5.21)

Control

Intervention

$1.04(.17)$

HPQ -

Presenteeism

864 Note.

865 iPBI= Irrational Performance Beliefs; CD-RISC $-10=$ Resilience; HCC $=$ Hair Cortisol Concentration;

866 DASS-21= Emotional Distress; HPQ = Presenteeism; $*=p<.05$.
$5.76 * \quad .16$

$.12 \quad .004$

$.75 \quad .02$

$.83 \quad .03$

$2.03 \quad .06$

$.45 \quad .01$

$1.22 \quad .04$ 


\section{Running Head: REBC and Fire Service}

Table 2.

Frequency, effect size (Cohen's d) and percentage change scores across cases between baseline and post-intervention, and between post-intervention and 3-month follow up time-points.

\begin{tabular}{|c|c|c|c|c|c|c|c|c|c|c|}
\hline \multicolumn{11}{|c|}{ Baseline - Post-Intervention } \\
\hline & \multicolumn{5}{|c|}{ Intervention } & \multicolumn{5}{|c|}{ Control } \\
\hline Measure & Change $(\%)$ & Effect size (SD) & Effect size & $\begin{array}{c}\text { Freq for } \\
\text { hypothesis }\end{array}$ & $\begin{array}{l}\text { Freq against } \\
\text { hypothesis }\end{array}$ & Change $(\%)$ & Effect size (SD) & $\begin{array}{l}\text { Effect size } \\
\text { range }\end{array}$ & $\begin{array}{c}\text { Freq for } \\
\text { hypothesis }\end{array}$ & $\begin{array}{c}\text { Freq against } \\
\text { hypothesis }\end{array}$ \\
\hline iPBI & -18.65 & $-1.41(1.49)$ & $-1.28-1.15$ & 15 & 3 & 1.29 & $0.04(0.77)$ & $-1.29-1.46$ & 7 & 9 \\
\hline $\begin{array}{c}\text { CD-RISC } \\
10\end{array}$ & 4.35 & $.24(.96)$ & $-1.49-1.74$ & 11 & 7 & 4.56 & $0.23(1.15)$ & $-2.96-1.82$ & 3 & 13 \\
\hline HCC & 35.50 & $-.31(1.08)$ & $-3.07-1.11$ & 11 & 7 & 90.54 & $-.11(1.40)$ & $-5.21-1.14$ & 11 & 5 \\
\hline Depression & N/A* & $-.38(.87)$ & $-2.13-2.13$ & 10 & 8 & N/A* & $-.59(1.09)$ & $-2.63-.75$ & 6 & 10 \\
\hline Anxiety & N/A* & $-.52(1.43)$ & $-4.33-1.24$ & 9 & 9 & N/A* & $-.73(1.19)$ & $-4.36-.48$ & 6 & 10 \\
\hline Stress & N/A* & $-.68(.94)$ & $-2.59-.97$ & 11 & 7 & N/A* & $-.17(1.31)$ & $-3.06-1.53$ & 10 & 6 \\
\hline HPQ & -1.22 & $-.23(1.46)$ & $-4.76-1.72$ & 9 & 9 & .25 & $-.18(1.44)$ & $-3.57-1.23$ & 11 & 6 \\
\hline \multicolumn{11}{|c|}{ Post-Intervention - 3-month follow-up } \\
\hline & \multicolumn{5}{|c|}{ Intervention } & \multicolumn{5}{|c|}{ Control } \\
\hline Measure & Change $(\%)$ & Effect size (SD) & $\begin{array}{l}\text { Effect size } \\
\text { range }\end{array}$ & $\begin{array}{c}\text { Freq for } \\
\text { hypothesis }\end{array}$ & $\begin{array}{l}\text { Freq against } \\
\text { hypothesis }\end{array}$ & Change $(\%)$ & Effect size (SD) & $\begin{array}{l}\text { Effect size } \\
\text { range }\end{array}$ & $\begin{array}{c}\text { Freq for } \\
\text { hypothesis }\end{array}$ & $\begin{array}{c}\text { Freq against } \\
\text { hypothesis }\end{array}$ \\
\hline iPBI & 4.50 & $0.17(.80)$ & $-1.28-1.62$ & 6 & 12 & -.93 & $-.09(.91)$ & $-2.43-1.88$ & 8 & 8 \\
\hline $\begin{array}{c}\text { CD-RISC } \\
10\end{array}$ & 3.53 & $.31(1.01)$ & $-1.85-2.16$ & 12 & 6 & 1.36 & $-.03(1.04)$ & $-2.45-1.47$ & 6 & 10 \\
\hline HCC & -7.58 & $-.83(1.47)$ & $-3.34-1.21$ & 12 & 6 & -19.31 & $-.98(1.54)$ & $-5.08-1.51$ & 3 & 13 \\
\hline Depression & N/A* & $.06(.62)$ & $-1.53-.51$ & 3 & 15 & N/A* & $-.04(1.11)$ & $-2.26-2.83$ & 12 & 4 \\
\hline Anxiety & N/A* & $-.36(1.30)$ & $-2.59-1.73$ & 5 & 13 & N/A* & $.49(1.01)$ & $-.82-1.63$ & 10 & 6 \\
\hline
\end{tabular}




\section{Running Head: REBC and Fire Service}

\begin{tabular}{|c|c|c|c|c|c|c|c|c|c|c|}
\hline Stress & N/A* & $.27(.74)$ & $-.78-1.17$ & 6 & 12 & N/A* & $-.07(1.14)$ & $-2.20-2.57$ & 5 & 11 \\
\hline HPQ & 9.65 & $0.73(1.43)$ & $-1.22-2.18$ & 12 & 6 & 2.81 & $.28(1.17)$ & $-2.41-2.90$ & 9 & 7 \\
\hline \multicolumn{11}{|c|}{ Baseline - 3-month follow-up } \\
\hline & \multicolumn{5}{|c|}{ Intervention } & \multicolumn{5}{|c|}{ Control } \\
\hline Measure & Change $(\%)$ & Effect size (SD) & $\begin{array}{l}\text { Effect size } \\
\text { range }\end{array}$ & $\begin{array}{c}\text { Freq for } \\
\text { hypothesis }\end{array}$ & $\begin{array}{c}\text { Freq against } \\
\text { hypothesis }\end{array}$ & Change $(\%)$ & Effect size (SD) & $\begin{array}{c}\text { Effect size } \\
\text { range }\end{array}$ & $\begin{array}{c}\text { Freq for } \\
\text { hypothesis }\end{array}$ & $\begin{array}{c}\text { Freq against } \\
\text { hypothesis }\end{array}$ \\
\hline iPBI & -26.67 & $-1.12(1.45)$ & $-3.25-1.27$ & 14 & 4 & -1.68 & $-0.05(.51)$ & $-1.05-.76$ & 7 & 9 \\
\hline $\begin{array}{c}\text { CD-RISC } \\
10\end{array}$ & 5.13 & $.49(1.24)$ & $-2.27-2.01$ & 12 & 6 & 2.75 & $.27(1.15)$ & $-2.75-2.50$ & 5 & 11 \\
\hline HCC & -194.66 & $-.60(1.41)$ & $-4.33-.38$ & 10 & 8 & -196.19 & $-.32(1.45)$ & $-5.73-.40$ & 6 & 10 \\
\hline Depression & N/A* & $-.33(.79)$ & $-1.85-.92$ & 10 & 8 & N/A* & $-.55(.98)$ & $-2.20-.74$ & 6 & 10 \\
\hline Anxiety & $\mathrm{N} / \mathrm{A}^{*}$ & $0.80(1.36)$ & $-4.60-1.32$ & 10 & 8 & $\mathrm{~N} / \mathrm{A}^{*}$ & $-.32(1.13)$ & $-3.53-1.01$ & 10 & 6 \\
\hline Stress & N/A* & $-.52(.98)$ & $-2.52-1.80$ & 9 & 9 & N/A* & $-.08(1.00)$ & $-.94-.95$ & 9 & 7 \\
\hline HPQ & 4.18 & $-.34(.98)$ & $-.98-1.96$ & 11 & 7 & -4.11 & $-.09(1.38)$ & $-2.66-2.04$ & 9 & 7 \\
\hline
\end{tabular}

Note. iPBI= Irrational Performance Beliefs; CD-RISC $-10=$ Resilience; HCC = Hair Cortisol Concentration; DASS-21= Emotional Distress; HPQ = Presenteeism *Percentage change scores for all three DASS-21 subscales were not obtained due a large number of participants scoring 0 at baseline and/or post-intervention timepoints. Frequency for and against hypothesis outlines the number of cases that were aligned to, or were contrary to the study hypotheses set out in the study introduction, and informed by previous research findings. 


\section{Running Head: REBC and Fire Service}

872

873 Table 3.

874 Mean, standard deviation, and frequency scores for social validation data collected on participants 875 within the intervention group $(n=10)$.

876

\begin{tabular}{|c|c|c|c|c|c|c|c|c|}
\hline Assessment Criteria & $\begin{array}{l}\text { Mean } \\
\text { (SD) }\end{array}$ & $\begin{array}{c}\text { Strongly } \\
\text { Disagree } \\
\text { (1) }\end{array}$ & $\begin{array}{c}\text { Disagree } \\
\text { (2) }\end{array}$ & $\begin{array}{c}\text { Somewhat } \\
\text { Disagree } \\
\text { (3) }\end{array}$ & $\begin{array}{c}\text { Neither } \\
\text { agree nor } \\
\text { disagree } \\
\text { (4) }\end{array}$ & $\begin{array}{c}\text { Somewhat } \\
\text { Agree } \\
\text { (5) }\end{array}$ & $\overline{\text { Agree }}$ & $\begin{array}{c}\text { Strongly } \\
\text { Agree } \\
\text { (7) }\end{array}$ \\
\hline Intervention was satisfactory. & $\begin{array}{l}5.89 \\
(.47)\end{array}$ & - & - & - & - & 1 & 8 & 1 \\
\hline Intervention was useful. & $\begin{array}{l}5.89 \\
(.94)\end{array}$ & - & - & - & 1 & 1 & 5 & 3 \\
\hline $\begin{array}{l}\text { Positive influence on general } \\
\text { wellbeing. }\end{array}$ & $\begin{array}{l}5.56 \\
(.82)\end{array}$ & - & - & - & 1 & 2 & 6 & 1 \\
\hline $\begin{array}{l}\text { Positive influence on } \\
\text { performance in workplace. }\end{array}$ & $\begin{array}{l}5.22 \\
(.82)\end{array}$ & - & - & - & 2 & 3 & 5 & - \\
\hline $\begin{array}{l}\text { Intervention application was } \\
\text { practical \& acceptable. }\end{array}$ & $\begin{array}{l}5.89 \\
(.32)\end{array}$ & - & - & - & - & 1 & 9 & - \\
\hline $\begin{array}{l}\text { Intervention was } \\
\text { understandable. }\end{array}$ & $\begin{array}{l}6.10 \\
(.32)\end{array}$ & - & - & - & - & - & 9 & 1 \\
\hline
\end{tabular}

877

878 